\title{
Distribuição da matéria orgânica e características de ácidos húmicos em solos com adição de resíduos de origem urbana ${ }^{(1)}$
}

\author{
Luciano Pasqualoto Canellas ${ }^{(2)}$, Gabriel de Araújo Santos ${ }^{(3)}$, Victor Marcos Rumjanek ${ }^{(4)}$, \\ Anselmo Alpande Moraes ${ }^{(4)}$ e Fernando Guridi(5)
}

\begin{abstract}
Resumo - O objetivo deste trabalho foi o de verificar possíveis alterações na distribuição das frações humificadas da matéria orgânica e das características estruturais de ácidos húmicos em amostras do horizonte superficial de dois solos (Latossolo Vermelho-Amarelo, e Argissolo Vermelho-Amarelo) que receberam o equivalente a $80 \mathrm{tha}^{-1}$ de composto de lixo urbano e lodo da estação de tratamento de esgoto. A distribuição das frações humificadas das amostras de solos com adição de resíduos urbanos foi alterada. Houve aumento do conteúdo de ácidos fúlvicos, implicando a diminuição da relação ácidos húmicos/ácidos fúlvicos e a qualidade do húmus do solo. Os ácidos húmicos estudados sofreram modificações nas suas características estruturais, principalmente com a diminuição do conteúdo de carbono em longas cadeias alquílicas e aumento no conteúdo de $\mathrm{C}$ atribuído a polissacarídeos incorporados às estruturas dos ácidos húmicos.
\end{abstract}

Termos para indexação: húmus, ácidos fúlvicos, propriedades físico-químicas do solo, espectrometria.

\section{Distribution of the organic matter and humic acid characteristic in soils with addition of residues of urban origin}

\begin{abstract}
The aim of this work was to determine potential changes in the distribution of humified fractions of the organic matter together with the structural features of humic acids in samples from the superficial horizon of two soils (Ultisol and Oxisol) to which 80 ton ha ${ }^{-1}$ of both compost of municipal residues and sewage sludge were added. An increase in the fulvic acids content was found which implies both a decrease in the humic/fulvic acid ratio and also in the quality of the humus in the soils. The humic acids studied underwent structural changes, mainly the decrease in the long alkyl chain content along with an increase in the $\mathrm{C}$ content related to polysaccharides incorporated to the humic acids.
\end{abstract}

Index terms: humus, fulvic acids, soil chemicophysical properties, spectrometry.

(1) Aceito para publicação em 7 de dezembro de 2000 Extraído da Tese de Doutorado apresentada pelo primeiro autor à Universidade Federal Rural do Rio de Janeiro (UFRRJ), Seropédica, RJ.

(2) Universidade Estadual do Norte Fluminense, Centro de Ciências e Tecnologias Agropecuárias, Av. Alberto Lamego, 2000, CEP 28015-620 Campos dos Goytacazes, RJ. Bolsista da Faperj. E-mail: canellas@uenf.br

(3)UFRRJ, Dep. de Solos, km 47 da antiga via RJ-SP, CEP 23851-970 Seropédica, RJ. Bolsista do CNPq. E-mail: gsantos@ufrrj.br

(4)UFRRJ, Dep. de Química. E-mail: rumjanek@ufrrj.br, amoraes@ufrrj.br

(5)Universidad Agraria de la Habana, Dep. de Química, San José de las Lajas, La Habana, Cuba. E-mail: fguridi@isch.edu.cu

\section{Introdução}

A utilização da matéria orgânica de origem industrial e urbana em terras agrícolas pode ser justificada pela necessidade de encontrar um destino apropriado para sua reciclagem. A matéria orgânica do solo e dos resíduos, do ponto de vista estritamente teórico, pode ser dividida em dois grandes compartimentos: um, composto pela fração não-humificada, representada pelos restos vegetais e animais pouco decompostos e pelos compostos orgânicos com categoria bioquímica definida (proteínas, açúcares, ceras, graxas, resinas), e outro, compartimento formado pelas substâncias humificadas. As substâncias húmicas são 
produto da intensa transformação dos resíduos orgânicos pela biomassa e polimerização dos compostos orgânicos (principalmente através de reações de condensação, demetilação e oxidação) até macromoléculas resistentes a degradação biológica (Santos \& Camargo, 1999). A matéria orgânica humificada do solo é composta por diferentes frações, de acordo com sua solubilidade. De modo geral, é aceito a distribuição das frações humificadas em três categorias: as huminas $(\mathrm{H})$ que representam a matéria orgânica intimamente ligada à fração mineral do solo, e por isso insolúvel; os ácidos fúlvicos (AF), que apresentam grande quantidade de grupamentos funcionais oxigenados e são solúveis tanto em meio ácido como básico, e os ácidos húmicos $(\mathrm{AH})$, insolúveis em meio fortemente ácido, pois, com a protonação dos grupamentos funcionais ocorre o colapso da estrutura e precipitação das macromoléculas. Os $\mathrm{AH}$ representam a fração reativa mais estável da matéria orgânica humificada.

O conteúdo relativo de cada fração da matéria orgânica é um indicativo da qualidade do húmus do solo (MacCallister \& Chuien, 2000). Alterações na qualidade do húmus em solos com adição de resíduos orgânicos de origem urbana estão ainda insuficientemente avaliadas.

As técnicas espectroscópicas permitem observar mudanças na conformação estrutural dos grupamentos funcionais da matéria orgânica. Com a espectroscopia de infravermelho (IV) é possível observar as oscilações do eixo (estiramentos) e do ângulo (deformações) das ligações entre os átomos de um grupamento funcional que apresente momento dipolo (diferença de polaridade entre os átomos de uma molécula), sendo útil na caracterização de grupamentos funcionais oxigenados da matéria orgânica.

$\mathrm{Na}$ espectroscopia de $\mathrm{RMN}{ }^{13} \mathrm{C}$, é observado um sinal de absorção de energia quando o ${ }^{13} \mathrm{C}$ é colocado na presença de um campo magnético e uma fonte de energia.

A freqüência de absorção do núcleo de ${ }^{13} \mathrm{C}$ é dependente do ambiente químico em torno do núcleo (como ambiente químico se entendem os diferentes grupamentos funcionais presentes na matéria orgânica), e a intensidade do sinal é proporcional à quantidade do grupamento funcional analisado.
O uso das técnicas espectroscópicas em conjunto permite o estudo das características estruturais da matéria orgânica.

O objetivo deste trabalho foi o de verificar possíveis alterações na distribuição das frações humificadas da matéria orgânica e das principais características estruturais de ácidos húmicos de dois solos que receberam adição de composto de lixo urbano e lodo da estação de tratamento de esgoto.

\section{Material e Métodos}

As características físico-químicas das amostras do horizonte superficial $(0-20 \mathrm{~cm})$ do Latossolo VermelhoAmarelo (LV) e do Argissolo Vermelho-Amarelo (AV), coletadas no Município de Piraí e Seropédica, respectivamente, ambos localizados no Rio de Janeiro, foram determinadas segundo Embrapa (1997); do composto de resíduos sólidos urbanos (Co) produzidos pela Companhia de Limpeza Urbana do Município do Rio de Janeiro (Comlurb), e de lodo da estação de tratamento de esgotos (L) da Companhia Estadual de Águas e Esgoto (CEDAE) do Rio de Janeiro, determinadas previamente nos trabalhos da Embrapa (1997), Mazur (1997) e Oliveira (1998).

Foram adicionados às amostras dos solos o equivalente a $80 \mathrm{t} \mathrm{ha}^{-1}$ dos resíduos orgânicos (Latossolo Vermelho-Amarelo + composto de resíduos sólidos urbanos: LVCo; Latossolo Vermelho-Amarelo + lodo da estação de tratamento de esgotos: LVL; Argissolo Vermelho-Amarelo + composto de resíduos sólidos urbanos: AVCo; e Argissolo Vermelho-Amarelo + lodo da estação de tratamento de esgotos: AVL).

Os tratamentos foram inteiramente casualizados em quatro repetições. Após 24 semanas de incubação em vasos com capacidade de $7 \mathrm{~kg}$, com teor de umidade mantido na capacidade de campo previamente determinada, de acordo com as condições experimentais descritas por Mazur (1997) e Oliveira (1998), e de um período de aproximadamente 80 semanas de armazenamento das amostras secas, foi realizado o fracionamento da matéria orgânica pelo método Kononova - Belchikova $(\mathrm{NaOH}+$ $\mathrm{Na}_{4} \mathrm{P}_{2} \mathrm{O}_{7}$ ambos $0,01 \mathrm{~mol} \mathrm{~L}^{-1}$ ), descrito em Kononova (1982), com quatro repetições por tratamento.

A dosagem de carbono nas frações foi realizada em três réplicas de laboratório por amostra. A análise estatística do fracionamento foi realizada dentro de cada tipo de solo isoladamente. Para a avaliação das características físico-químicas dos ácidos húmicos $(\mathrm{AH})$, as quatro repetições dos tratamentos foram reunidas, e a extração foi realizada com $\mathrm{NaOH} 0,5 \mathrm{~mol} \mathrm{~L}^{-1}$, na razão solvente: solo de 
1:10 (v:v) em atmosfera inerte de $\mathrm{N}_{2}$. A separação dos $\mathrm{AH}$ foi conseguida com o abaixamento do $\mathrm{pH}$ da solução até $1,0 \mathrm{com} \mathrm{HCl} 6 \mathrm{~mol} \mathrm{~L}^{-1}$. A redissolução e precipitação foi repetida três vezes. Em seguida, foram adicionadas $200 \mathrm{~mL}$ de solução aquosa diluída de $\mathrm{HF}$ e $\mathrm{HCl}$ (preparada com $5 \mathrm{~mL}$ de $\mathrm{HCl}$ concentrado, e $5 \mathrm{~mL}$ de $\mathrm{HF}$ concentrado e volume de solução completado para $1 \mathrm{~L}$ com água deionizada), e agitou-se a amostra por oito horas. $\mathrm{Os} \mathrm{AH}$ foram lavados com água até teste negativo com $\mathrm{AgNO}_{3} \mathrm{e}$ secados por liofilização. Após o processo de extração e purificação, os $\mathrm{AH}$ foram caracterizados pelas seguintes análises:

A determinação da composição elementar foi realizada com analisador elementar automático Perkin Elmer 2400, em amostras de $1 \mathrm{mg}$ de AH em duplicata. O conteúdo de $\mathrm{O}$ foi determinado por diferença, descontado o teor de cinzas obtido com a incineração de três amostras dos $\mathrm{AH}$ por tratamento em mufla a $700^{\circ} \mathrm{C}$. A relação $E_{4} / E_{6}$ foi obtida por meio da razão da absorvância de uma solução de $\mathrm{AH}(4 \mathrm{mg}$ em $10 \mathrm{~mL}$ de $\mathrm{NaHCO}_{3} \quad 0,05 \mathrm{~mol} \mathrm{~L}^{-1}$ ) em $465 \mathrm{~nm}$ e $665 \mathrm{~nm}$ num espectrofotômetro Beckman mod. B.

A espectroscopia na região do infravermelho (IV) dos AH foi realizada na faixa de $600 \mathrm{~cm}^{-1}$ a $4.000 \mathrm{~cm}^{-1}$, utilizando-se pastilhas com $1 \mathrm{mg}$ de $\mathrm{AH}$ em $100 \mathrm{mg}$ de $\mathrm{KBr}$, num aparelho Perkin Elmer 1420.

Os espectros de $\mathrm{RMN}{ }^{13} \mathrm{C}$ foram obtidos com $150 \mathrm{mg}$ de $\mathrm{AH}$ em $1 \mathrm{~mL}$ de $\mathrm{NaOH}$ 0,5 mol L-1 (1:1 $\left.\mathrm{H}_{2} \mathrm{O}: \mathrm{D}_{2} \mathrm{O}\right)$, num aparelho Bruker AC-200, com freqüência de 50,30 MHz para ${ }^{13} \mathrm{C}$, utilizando a seqüencia INVGATE (desacoplamento inverso), com pulso de $90^{\circ}$, tempo de aquisição de $0,2 \mathrm{~s}, 16 \mathrm{~K}$ de tamanho e média de 200.000 transientes por amostra. Todos os espectros foram obtidos à temperatura ambiente, e os deslocamentos químicos foram observados a partir do deslocamento químico do tetrametilsilano (TMS) usado como referência.

\section{Resultados e Discussão}

Houve aumento dos teores de $\mathrm{Ca}$ e $\mathrm{Mg}$ nas amostras que receberam os resíduos (Tabela 1). Notouse, também, aumento da relação $\mathrm{Ca} / \mathrm{Mg}$ de 0,5 para 1,36 e 1,5 nas amostras do LV, e de 1 para 1,87 e 2,31 nas amostras de AV, com adição de composto e de lodo, respectivamente. Os efeitos da adição dos resíduos orgânicos sobre os parâmetros químicos da fertilidade desses solos, inclusive teores de metais pesados, foram estudados por Mazur (1997) e Oliveira (1998)
A maior parte da matéria orgânica dos dois solos é constituída pela fração não humificada, chamada de matéria orgânica leve (Tabela 2). As frações humificadas representam 43 e $45 \%$ do carbono orgânico do $\mathrm{LV}$ e $\mathrm{AV}$, respectivamente. No $\mathrm{LV}$, houve um equilíbrio maior entre as frações humificadas reativas, verificado pelo valor da relação $\mathrm{AH} / \mathrm{AF}$ próximo da unidade. A relação $\mathrm{AH} / \mathrm{AF}$, em ambos os resíduos, está distante de 1 , conferindo ao húmus caráter fúlvico (Ortega, 1983). A predominância de AF sobre os AH é indicativo de características desfavoráveis ao húmus dos resíduos orgânicos em relação ao húmus do solo (Garcés, 1987; Moreno, 1996). A adição dos resíduos no solo promoveu modificações na distribuição das frações humificadas da matéria orgânica no LV, ocorrendo aumento significativo no conteúdo de substâncias húmicas. Nas amostras de AV, a distribuição da fração humificada não foi alterada com a adição dos resíduos orgânicos. O maior conteúdo de argila no LV (Tabela 1) pode favorecer a estabilização da matéria orgânica humificada por meio da formação de complexos organo-minerais, tornando a matéria orgânica relativamente mais protegida da degradação microbiana. O conteúdo de $\mathrm{AH}$ não foi significativamente alterado com a adição dos resíduos nas amostras dos solos estudados. De acordo com Orlov (1998), o aumento no conteúdo de ácidos húmicos pode ser um indicador da melhoria da qualidade do húmus do solo ou do incremento da atividade biológica que promove a síntese de substâncias húmicas mais condensadas. $\mathrm{O}$ aumento no conteúdo de substâncias húmicas observado nas amostras de LV com adição dos resíduos foi às custas do incremento, principalmente, da fração humina (aumento de 63 e 94\% com adição de lodo e composto, respectivamente), e também dos ácidos fúlvicos com a adição de composto (aumento de 192\%). Neste caso, a transformação da matéria orgânica não privilegia a formação de substâncias alcalino-solúveis condensadas, e, provavelmente, favorece a estabilização direta, via interação com a fração mineral e sua dissociação em moléculas menos condensadas. Tal fenômeno foi observado por Dabin (1981), em solos tropicais da África. O fracionamento químico da matéria orgânica humificada reflete somente o balanço líquido quantitativo da matéria orgânica. Embora não se tenha 
observado mudanças quantitativas no conteúdo de ácidos húmicos, foram constatadas alterações nas suas características químicas. Os AH de LV apresentam maior conteúdo de $\mathrm{C}$ e menor de $\mathrm{O}$ que os $\mathrm{AH}$ de amostras com a adição de resíduos (Tabela 3). O mesmo fenômeno foi observado em relação ao conteúdo de $\mathrm{C}$ do $\mathrm{AV}$. $\mathrm{O}$ conteúdo de $\mathrm{N}$ dos resíduos orgânicos de origem urbana é elevado (Tabela 1), e

Tabela 1. Propriedades físico-químicas do composto de resíduos sólidos urbanos (Composto), de lodo da estação de tratamento de esgotos (Lodo) e das amostras do horizonte superficial dos Latossolo Vermelho-Amarelo e Argissolo Vermelho-Amarelo (Solo testemunha) e dos solos com adição dos resíduos (Solo + composto e Solo + lodo).

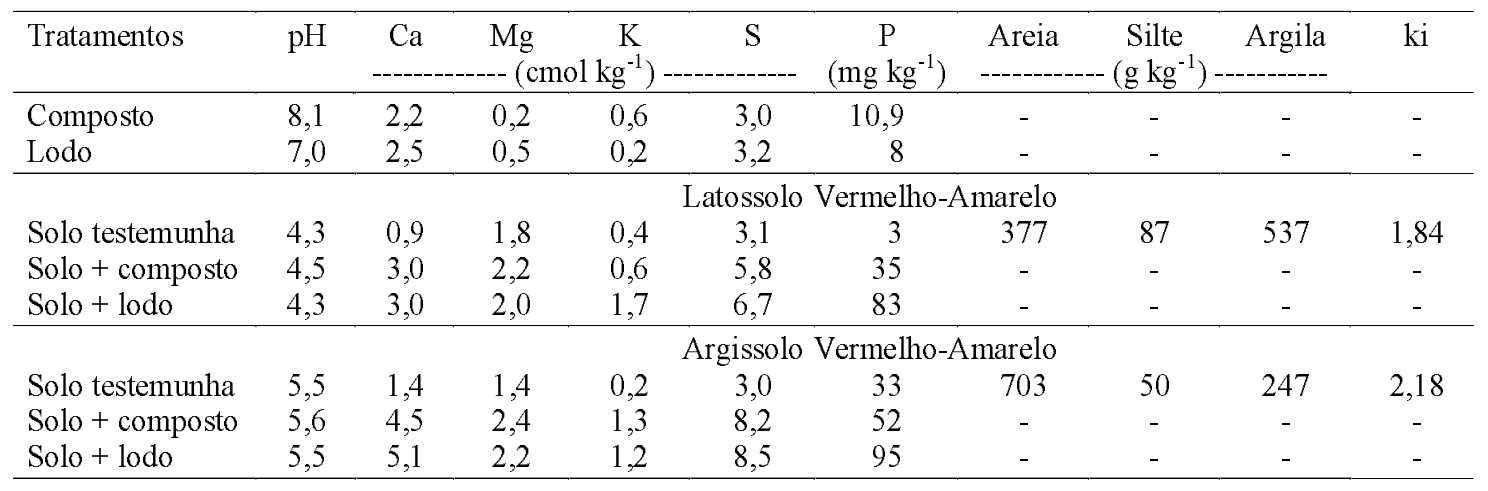

Tabela 2. Fracionamento da matéria orgânica do composto de resíduos sólidos urbanos (Composto), de lodo da estação de tratamento de esgotos (Lodo) e das amostras do horizonte superficial dos Latossolo Vermelho-Amarelo e Argilossolo Vermelho-Amarelo (Solo testemunha) e dos solos com adição dos resíduos (Solo + composto e Solo + lodo) ${ }^{(1)}$.

\begin{tabular}{|c|c|c|c|c|c|c|c|c|}
\hline \multirow[t]{3}{*}{ Tratamentos } & \multicolumn{8}{|c|}{ Fracionamento da matéria orgânica } \\
\hline & $\mathrm{C}$ & $\mathrm{N}$ & $\mathrm{C} / \mathrm{N}$ & $\begin{array}{l}\text { Substâncias } \\
\text { húmicas }\end{array}$ & Huminas & $\begin{array}{l}\text { Ácidos } \\
\text { fúlvicos } \\
(\mathrm{AF})\end{array}$ & $\begin{array}{l}\text { Ácidos } \\
\text { húmicos } \\
(\mathrm{AH})\end{array}$ & $\mathrm{AH} / \mathrm{AF}^{(2)}$ \\
\hline & \multicolumn{8}{|c|}{ 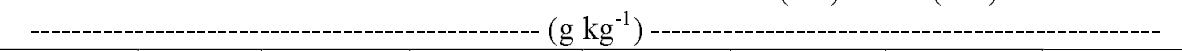 } \\
\hline Composto & 222,7 & 13,0 & 15,5 & 154,4 & 107,0 & 42,2 & 5,2 & 0,12 \\
\hline \multirow{2}{*}{ Lodo } & 186,0 & 21,5 & 8,6 & 143,0 & 94,7 & 39,4 & 9,6 & 0,24 \\
\hline & \multicolumn{8}{|c|}{ Latossolo Vermelho-Amarelo } \\
\hline Solo testemunha & 24,4 & 2,3 & 11,0 & $10,5 \mathrm{a}$ & $7,6 a$ & $1,4 b$ & 1,5 & $1,00 \mathrm{a}$ \\
\hline Solo + composto & 27,6 & 2,8 & 9,8 & $20,6 b$ & $14,8 \mathrm{~b}$ & $4,1 \mathrm{a}$ & 1,7 & $0,41 b$ \\
\hline Solo + lodo & 22,1 & 3,2 & 7,0 & $16,1 b$ & $12,4 \mathrm{~b}$ & $2,0 \mathrm{~b}$ & 1,7 & $0,85 \mathrm{ab}$ \\
\hline Média & $24,70^{\mathrm{ns}}$ & $2,76^{\mathrm{ns}}$ & $9,26^{\mathrm{ns}}$ & $15,93 * *$ & $11,60 * *$ & $2,50 * *$ & $1,63^{\mathrm{ns}}$ & $0,75 * *$ \\
\hline \multirow[t]{2}{*}{ Desvio padrão } & 2,81 & 0,69 & 2,05 & 5,15 & 3,42 & 1,39 & 0,11 & 0,25 \\
\hline & \multicolumn{8}{|c|}{ Argissolo Vermelho-Amarelo } \\
\hline Solo testemunha & 16,0 & 1,0 & 16,0 & 7,3 & 5,2 & 1,3 & 0,8 & 0,60 \\
\hline Solo + composto & 14,7 & 1,3 & 11,3 & 8,7 & 6,2 & 1,7 & 0,8 & 0,47 \\
\hline Solo + lodo & 13,7 & 1,5 & 9,1 & 7,9 & 5,8 & 1,4 & 0,7 & 0,50 \\
\hline Média & $14,8^{\mathrm{ns}}$ & $1,26^{\mathrm{ns}}$ & $12,3 * *$ & $7,9 * *$ & $5,73^{\mathrm{ns}}$ & $1,14^{\mathrm{ns}}$ & $0,76^{\mathrm{ns}}$ & $0,52 * *$ \\
\hline Desvio padrão & 1,77 & 0,43 & 3,52 & 1,69 & 1,20 & 0,48 & 0,23 & 0,18 \\
\hline
\end{tabular}

${ }^{(1)}$ Médias seguidas de letras iguais, na coluna, não diferem estatisticamente entre si pelo teste de Tukey $(\mathrm{p}<0,005)$. (2) AH/AF: relação ácidos húmicos: ácidos

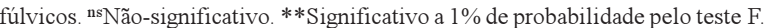


os $\mathrm{AH}$ desses resíduos apresentam valores de $\mathrm{N}$ um pouco acima da faixa observada para $\mathrm{AH}$ de solos (normalmente entre 0,8 e 4,3\% segundo Stevenson, 1994). No LV, a adição de Co não alterou o conteúdo de $\mathrm{N}$ dos $\mathrm{AH}$. Por outro lado, a adição de lodo reduziu esse valor em cerca de $10 \%$. Tal fato, se deve, provavelmente, ao efeito conhecido como mineralização secundária da matéria orgânica. $\mathrm{O}$ aporte de matéria orgânica ao solo promove maior atividade da biomassa microbiana, que utiliza, na obtenção de energia, fontes de $\mathrm{C}$ já existentes no solo (Kononova, 1982), uma vez que foi observado decréscimo no conteúdo de $\mathrm{C}$ nos $\mathrm{AH}$ das amostras de $\mathrm{LV}$ com a adição dos resíduos. Nos $\mathrm{AH}$ isolados das amostras do AV incubado com os diferentes resíduos, verificaram-se incrementos de $\mathrm{N}$ na ordem de 25 e $7 \%$ com adição de composto e lodo, respectivamente. A preservação de $\mathrm{N}$ nos $\mathrm{AH}$, por meio da proteção exercida pela interação com a fração mineral não pode ser descartada, mas o conteúdo de argilas neste solo é menor do que no Latossolo, bem como seu grau de intemperismo, avaliado pelo maior valor $\mathrm{Ki}$ (Tabela 1). Por outro lado, o aumento do conteúdo de $\mathrm{N}$ é um indicador do processo de evolução dos AH, de acordo com Kumada (1987), evidenciando a presença de transformações químicas mais intensas no $\mathrm{AV}$, decorrentes da humificação nos $\mathrm{AH}$ isolados das amostras com adição dos resíduos. Os menores valores da relação $\mathrm{C} / \mathrm{N}$ nos $\mathrm{AH}$ do $\mathrm{AV}$ com a adição dos resíduos orgânicos indicam a maior estabilidade química desta fração humificada. Os valores de $\mathrm{O}$ encontrados em todos $\mathrm{AH}$ estudados estão muito próximos ou acima da faixa normalmente observada em relação a ácidos húmicos de origem pedogênica, entre 32,8 e 38,3\% (Stevenson, 1994). Nota-se que com a adição de Co há uma tendência de diminuição no conteúdo de $\mathrm{O}$ nos $\mathrm{AH}$ de ambos os solos, e que com a adição de L ocorre, ao contrário, um aumento nesses valores. A relação molar atômica $\mathrm{H} / \mathrm{C}$ é uma medida indireta das características estruturais dos $\mathrm{AH}$. Quanto menor o valor da relação, mais acentuado é o caráter aromático do carbono. Os menores valores da relação $\mathrm{H} / \mathrm{C}$ foram encontrados no $\mathrm{LV}$ com a adição dos resíduos orgânicos.

Os valores obtidos da relação $\mathrm{E}_{4} / \mathrm{E}_{6}$ dos $\mathrm{AH}$ estão próximos da faixa normalmente encontrada nos $\mathrm{AH}$, entre 3 e 5 (Chen et al., 1977), o que sugere um elevado grau de condensação dos $\mathrm{AH}$, uma vez que a magnitude da relação está inversamente relacionada com o tamanho molecular e o conteúdo de $\mathrm{C}$ aromáticos (Ghosh \& Schnitzer, 1979).

Na Figura 1, são apresentados os espectros na região do IV dos AH. As possíveis atribuições das bandas de absorção foram realizadas de acordo com Colthup et al. (1964), Garcés (1987), Bloom \& Leenheer (1989) e Stevenson (1994). Nos espectros de IV observa-se uma banda de absorção bem definida na região de $3.694 \mathrm{~cm}^{-1}$, que deve corresponder a vibrações de $\mathrm{O}-\mathrm{H}$ ou $\mathrm{N}-\mathrm{H}$ livres. Na região de 3.600 a $3.070 \mathrm{~cm}^{-1}$, é observada uma banda de absorção larga atribuída a $\mathrm{O}-\mathrm{H}$ em ligação $\mathrm{H}$, e, possivelmente, grupos N-H (Nakanishi, 1962). A presença de pequenos ombros na região de $3.080 \mathrm{~cm}^{-1} \mathrm{em}$ todos os espectros sugere a presença de estruturas aromáticas. Na região de $2.900 \mathrm{~cm}^{-1}$, foram observadas bandas de absorção, devido à presença de grupos

Tabela 3. Composição elementar ( \pm erro-padrão da média), relações $\mathrm{C} / \mathrm{N}, \mathrm{H} / \mathrm{C}$ e $\mathrm{O} / \mathrm{C}$, teor de cinzas e relação $\mathrm{E}_{4} / \mathrm{E}_{6}$ dos ácidos húmicos extraídos das amostras de solos sem adição de resíduos, Latossolo Vermelho Amarelo (LV) e Argissolo Vermelho Amarelo (AV), e com adição de composto de resíduo sólido urbano (LVCo e AVCo) e de lodo da estação de tratamento de esgoto (LVL e AVL).

\begin{tabular}{lcccccccc}
\hline Parâmetro & LV & AV & Co & L & LVCo & LVL & AVCo & AVL \\
\hline C $(\%)$ & $53,1 \pm 0,3$ & $57,95 \pm 0,1$ & $50,7 \pm 0,4$ & $54,0 \pm 0,2$ & $51,8 \pm 0,58$ & $50,9 \pm 0,33$ & $55,85 \pm 0,41$ & $48,75 \pm 0,33$ \\
H $(\%)$ & $4,70 \pm 0,07$ & $5,04 \pm 0,09$ & $6,2 \pm 0,01$ & $7,0 \pm 0,04$ & $3,96 \pm 0,07$ & $2,53 \pm 0,05$ & $5,00 \pm 0,03$ & $4,63 \pm 0,04$ \\
N $(\%)$ & $2,27 \pm 0,06$ & $2,31 \pm 0,04$ & $6,0 \pm 0,01$ & $4,9 \pm 0,02$ & $2,26 \pm 0,07$ & $2,05 \pm 0,06$ & $2,88 \pm 0,1$ & $2,48 \pm 0,08$ \\
O $(\%)$ & $35,92 \pm 1,11$ & $34,7 \pm 2,15$ & $37,1 \pm 1,02$ & $34,1 \pm 1,20$ & $38,48 \pm 1,01$ & $40,67 \pm 1,30$ & $32,3 \pm 1,31$ & $39,9 \pm 1,15$ \\
$\mathrm{C} / \mathrm{N}$ & 23,34 & 25,08 & 9,8 & 12,8 & 22,86 & 24,82 & 19,39 & 19,65 \\
$\mathrm{H} / \mathrm{C}$ & 1,06 & 1,04 & 1,42 & 1,5 & 0,92 & 0,59 & 1,07 & 1,31 \\
$\mathrm{O} / \mathrm{C}$ & 0,51 & 0,45 & 0,54 & 0,47 & 0,55 & 0,59 & 0,43 & 0,61 \\
Cinzas $(\%)$ & $4,0 \pm 1,01$ & 5,31 & - & - & $3,5 \pm 0,78$ & $3,85 \pm 0,91$ & 3,97 & 4,23 \\
$\mathrm{E}_{4} / \mathrm{E}_{6}$ & 2,8 & 2,3 & 4,1 & 4,3 & 2,7 & 3,9 & 2,4 & 4,0 \\
\hline
\end{tabular}


$\mathrm{C}-\mathrm{H}\left(\mathrm{CH}_{3}\right)$ confirmado pela absorção em $1.456 \mathrm{~cm}^{-1}$. No LV, é observada uma absorção adicional em $2.848 \mathrm{~cm}^{-1} \mathrm{e}$ nas amostras de AH de AV com e sem adição de resíduos em $2.847 \mathrm{~cm}^{-1}$. Essa absorção tam-
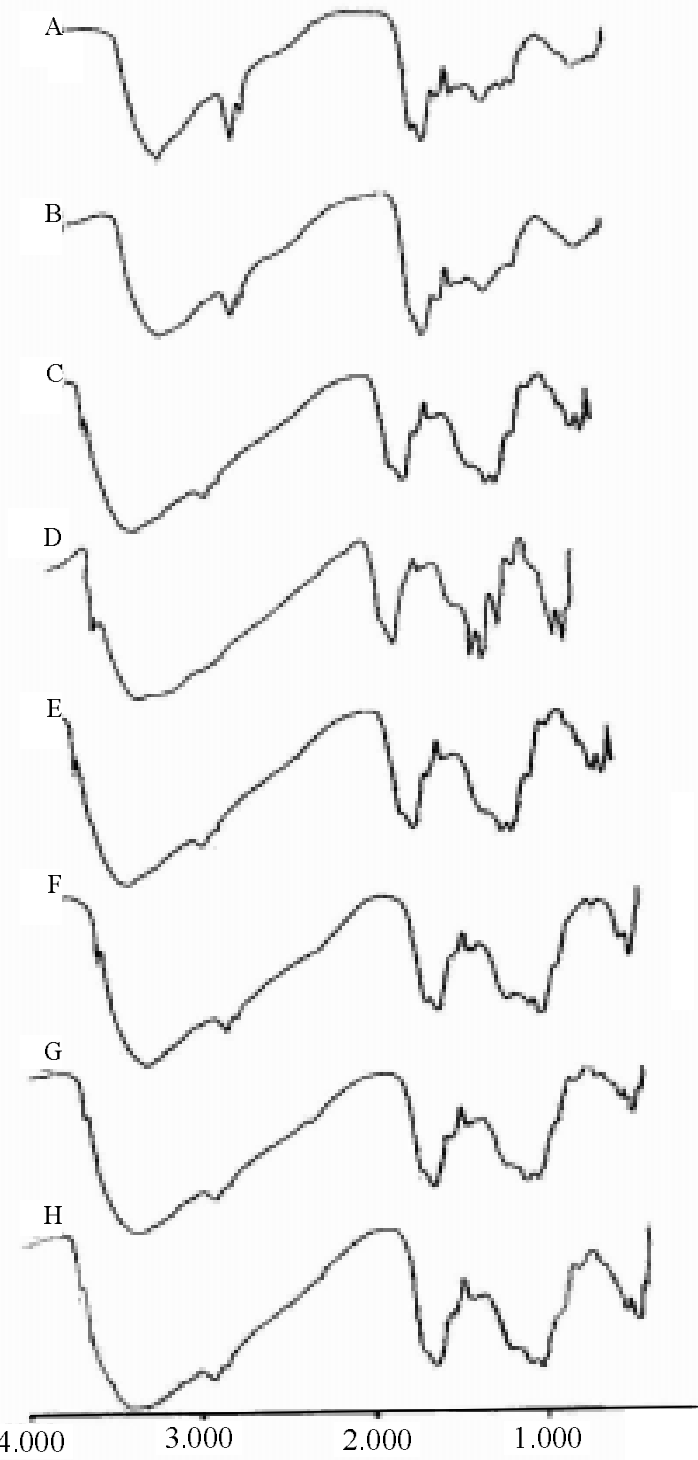

Figura 1. Espectros de infravermelho $\left(\mathrm{cm}^{-1}\right)$ dos ácidos húmicos de composto de resíduos sólidos urbanos (A), de lodo da estação de tratamento de esgotos (B), de Latossolo Vermelho-Amarelo sem (C) e com adição dos resíduos (D e E) e de Argilossolo Vermelho-Amarelo sem (F) e com adição dos resíduos $(\mathrm{G}$ e $\mathrm{H})$. bém é atribuída a C-H alifático, só que característico de grupos $\mathrm{CH}_{2}$. As absorções ao redor de $1.700 \mathrm{~cm}^{-1}$ correspondem a vibrações de grupos $\mathrm{C}=\mathrm{O}$ de cetonas, quinonas e grupos $\mathrm{COOH}$ (Silverstein et al., 1994). Nas amostras de AH do LV sem a adição de resíduos, o centro desta absorção está deslocado para uma região de maior energia em relação aos $\mathrm{AH}$ das amostras com adição de resíduos, o que indica a possibilidade, nos AH com adição de resíduos, da presença de uma conjugação maior. Fenômeno contrário é observado nos $\mathrm{AV}$.

A incorporação de resíduos aumenta o conteúdo de $\mathrm{O}$ nos LV e diminui no caso do AVCo (Tabela 2). Nas amostras de AH extraídos do AV e do AV com adição de Co e L foram observadas absorções muito fracas em $2.347 \mathrm{~cm}^{-1}, 2.369 \mathrm{~cm}^{-1} \mathrm{e} 2.847 \mathrm{~cm}^{-1}$, respectivamente. Absorções semelhantes foram encontradas por Garcés (1987) e atribuídas a vibrações de grupos $\mathrm{COOH}$ não-ionizados. Este fato concorda com o maior conteúdo de cinzas nos AV. É observada uma banda de absorção em torno 1.646 a $1.635 \mathrm{~cm}^{-1}$, que pode ser atribuída à presença do íon carboxilato (deformação axial assimétrica). A presença de uma pequena banda de absorção em $1.543 \mathrm{~cm}^{-1}$ nos LV pode sugerir, também, de acordo com Colthup et al. (1964), a presença de amidas (bandas I e II, respectivamente). As bandas de absorção em 1.435, $1.348 \mathrm{e}$ $1.217 \mathrm{~cm}^{-1}$ são indicativo da presença de grupos $\mathrm{COO}^{-}, \mathrm{C}-\mathrm{O}$ e $\mathrm{OH}$ de $\mathrm{COOH}$ (Nakanishi, 1962; Colthup et al., 1964; Silverstein et al., 1994). Em todos os espectros aparece uma absorção bem definida em $1.100 \mathrm{~cm}^{-1}$. Garcés (1987) atribuiu este sinal à presença de grupos $\mathrm{COO}$ ligados a cátions metálicos. A presença de um teor elevado de cinzas nas amostras de $\mathrm{AH}$ (Tabela 2) sugere que este sinal corresponda a impurezas na amostra, e que essa absorção pode servir como um índice qualitativo de pureza de substâncias húmicas. Também está presente em todos os espectros um sinal de absorção centrado em $1.038 \mathrm{~cm}^{-1}$, que pode ser atribuído à presença de carboidratos nos $\mathrm{AH}$. Os sinais são mais intensos nas amostras de AH extraídas de solos que receberam a adição de resíduos. As absorções compreendidas entre $900 \mathrm{e} 500 \mathrm{~cm}^{-1}$ geralmente são atribuídas à presença de impurezas minerais nas substâncias húmicas (Bloom \& Leenheer, 1989). 
Os espectros de RMN ${ }^{13} \mathrm{C}$ possuem, de modo geral, a mesma feição em todas as amostras de $\mathrm{AH}$, porém com intensidades e áreas dos sinais diferentes em cada amostra (Figura 2). As possíveis atribuições dos sinais foram baseadas no conhecimento acumulado pela espectroscopia de RMN ${ }^{13} \mathrm{C}$ em solos e ácidos húmicos nos trabalhos de Wilson (1984, 1987), Schnitzer \& Preston (1986), Preston et al. (1994a, 1994b), Preston (1996), Köbel-Knaber (1997), Schulten \& Schnitzer (1997), Veeman (1997), Zech et al. (1997) (Tabela 4).

A utilização da técnica de desacoplamento inverso de hidrogênio (INVGATE) permitiu a análise quantitativa dos espectros de RMN de ${ }^{13} \mathrm{C}$, que foram divididos em cinco áreas de absorção: $\delta_{\mathrm{C}} 0-48$ (CH alifáticos), $\delta_{\mathrm{C}}$ 50-70 (C monooxigenados e nitrogenados), $\delta_{\mathrm{C}} 100$ (C anomérico), $\delta_{\mathrm{C}} 110-140$ (C aromáticos) e $\delta_{\mathrm{C}} 160-185$ (C carboxílicos).

Em comparação com as amostras de $\mathrm{AH}$ do solo que não receberam adição de resíduos, os $\mathrm{AH}$ extraídos das amostras incubadas apresentaram menor conteúdo de $\mathrm{C}$ alquílico (Tabela 5). A adição de Co no LV promoveu decréscimo nas áreas integradas correspondentes a $\mathrm{C}$ monoxigenados e nitrogenados. Entretanto, aumentou consideravelmente a quantidade de $\mathrm{C}$ anomérico na estrutura dos $\mathrm{AH}$, indicando, assim, ação da atividade microbiana sobre a matéria orgânica humificada.

A adição de lodo de esgoto no Latossolo promove alterações diferentes na estrutura dos AH. Ocorre aumento na região de 50 a 70 ppm, e não próximo a $100 \mathrm{ppm}$.

O grau de aromaticidade das amostras de LV permanece praticamente inalterado, mostrando incorporação de estruturas condensadas nos $\mathrm{AH}$. Com a espectroscopia de $\mathrm{RMN}{ }^{13} \mathrm{C}$ foi possível observar que o acréscimo de $\mathrm{O}$ na composição elementar dos $\mathrm{AH}$ obtidos de LV com a adição dos resíduos orgânicos é decorrente do aumento de polissacarídeos nos AH, pois na região de $\mathrm{COOH}$ há nítida diminuição de valor indicando um processo de descarboxilação dos LV.

A adição de matéria orgânica de origem urbana sobre as amostras de AV também promoveu alterações na estrutura dos $\mathrm{AH}$. Na região de $\mathrm{C}$ saturados totais ( 0 a $120 \mathrm{ppm}$ ), as alterações foram praticamente as mesmas dos $\mathrm{AH}$ extraídos de LV.

$A$ adição de Co promoveu incremento maior na região de $\mathrm{C}$ anomérico, e com a adição de $\mathrm{L}$, o maior

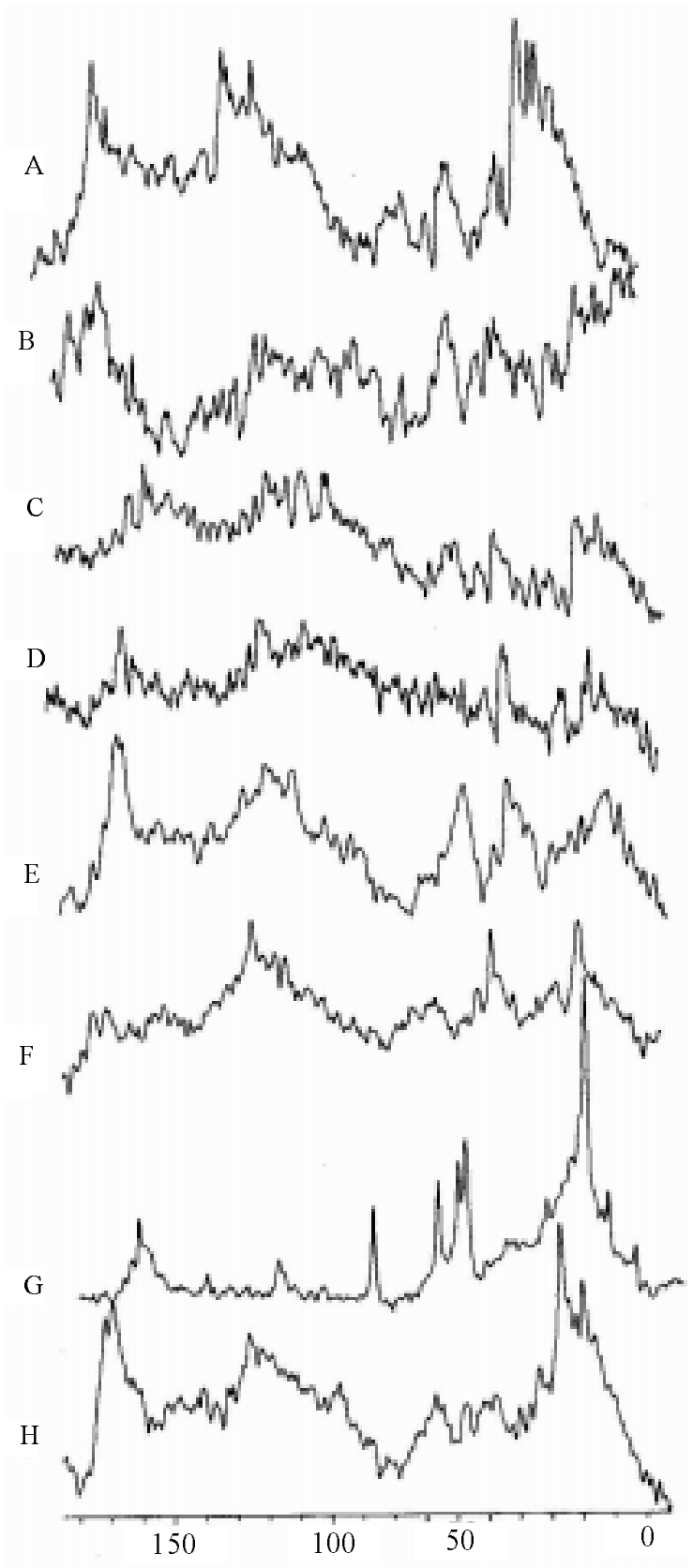

Figura 2. Espectros de $\mathrm{RMN}{ }^{13} \mathrm{C}$ (ppm) dos ácidos húmicos isolados de Argissolo (A), Argissolo com adição de composto (B) e lodo (C), de Latossolo (D), Latossolo com adição de composto (E) e de lodo (F) e de composto de resíduos sólidos urbanos $(\mathrm{G})$ e lodo da estação de tratamento de esgoto $(\mathrm{H})$.

Pesq. agropec. bras., Brasília, v. 36, n. 12, p. 1529-1538, dez. 2001 
Tabela 4. Principais atribuições dos espectros de $\mathrm{RMN}^{13} \mathrm{C}$ dos ácidos húmicos $(\mathrm{AH})$ estudados.

\begin{tabular}{lll}
\hline$\delta_{\mathrm{C}}(\mathrm{em} \mathrm{ppm})$ & Atribuições $^{(1)}$ & Fórmula química geral \\
\hline 15,$8 ; 21$ & Grupos metilas & $-\mathrm{CH}_{3}$ \\
$27,30,38,40$ & Grupos metilenos & $-\left(\mathrm{CH}_{2}\right)_{\mathrm{n}}$ \\
55 & Grupos metoxilas & $\mathrm{O}-\mathrm{CH}_{3}$ \\
61 & C em polissacarídeos & $\mathrm{CH}_{2}-\mathrm{OH}$ \\
74 & C alifático substituído por grupos - $\mathrm{OH}$ ou $-\mathrm{O}-\mathrm{ou}$ & $\mathrm{CH}-\mathrm{OH}$ \\
& carbono mononitrogenado & $-\mathrm{CH}-\mathrm{NH}_{2}$ \\
$99-106$ & C duplamente oxigenados & $\mathrm{H}$ \\
& & $-\mathrm{C}$ \\
& & \\
$115-131$ & C aromáticos protonados ou sem substituição por & $\mathrm{CH}$ em aromáticos; \\
$152-161$ & elemento forte doador de elétrons & $\mathrm{C}-\mathrm{ARIL}-\mathrm{C}-\mathrm{CH}(\mathrm{R})_{2}$ \\
$169 \mathrm{e} 174$ & C acromáticos substituídos por heteroátomo; fenóis & ARIL-C-OCH $;$ - ARIL-CH $-\mathrm{OH}$ \\
\hline
\end{tabular}

(1)As possíveis atribuições foram realizadas de acordo com os trabalhos de Wilson (1984, 1987), Schnitzer \& Preston (1986), Preston et. al (1994a, 1994b), Preston (1996), Köbel-Knaber (1997), Schulten \& Schnitzer (1997), Veeman (1997) e Zech et al. (1997)

Tabela 5. Integração das áreas de absorção dos espectros de $\mathrm{RMN}$ de ${ }^{13} \mathrm{C}$ das amostras de ácidos húmicos (AH) de Latossolo Vermelho-Amarelo (LV) e Argilossolo Vermelho-Amarelo (AV) com e sem adição de composto de resíduos sólidos de origem urbana (Co) e lodo da estação de tratamento de esgotos (L).

\begin{tabular}{lccccccrr}
\hline$\delta_{\mathrm{C}(\mathrm{ppm})}$ & Co & L & LV & LVCo & LVL & AV & AVCo & AVL \\
\hline $0-48$ & 43,8 & 36,4 & 25,9 & 21,1 & 23,2 & 38,4 & 19,2 & 27,4 \\
$50-70$ & 10,2 & 19,1 & 23,4 & 15,8 & 27,8 & 8,9 & 8,7 & 13,0 \\
100 & 9,3 & $\mathrm{~ns}$ & $\mathrm{~ns}$ & 15,9 & $\mathrm{~ns}$ & $\mathrm{~ns}$ & 19,2 & 8,6 \\
$120-140$ & 29,3 & 33,3 & 32,3 & 34,0 & 33,7 & 33,7 & 33,8 & 32,4 \\
$160-185$ & 8,7 & 11,2 & 18,3 & 12,9 & 15,2 & 18,9 & 18,9 & 18,6 \\
$\mathrm{GA}^{(1)}$ & 32,0 & 37,5 & 39,6 & 39,2 & 39,8 & 41,6 & 41,6 & 39,8 \\
\hline
\end{tabular}

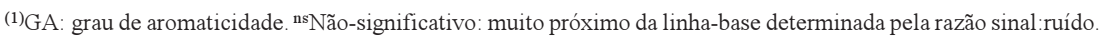

acréscimo de $\mathrm{C}$ foi na região de 50 a 70 ppm. A adição de $\mathrm{L}$ promove um pequeno decréscimo na quantidade de $\mathrm{C}$ aromáticos, e, conseqüentemente, no grau de aromaticidade, o que não era esperado por motivo da maior estabilidade deste tipo de resíduo orgânico.

Esta análise é corroborada pelo pequeno aumento ocorrido na relação $\mathrm{H} / \mathrm{C}$ nestes $\mathrm{AH}$. Isto é indicativo de que existe uma certa seletividade nas alterações estruturais dos AH. Com a adição de L no $\mathrm{AV}$ (que apresenta textura mais grosseira), pode ter ocorrido acréscimo de $\mathrm{C}$, por causa dos polissacarídeos produzidos pela atividade biológica e incorporados nas estruturas dos $\mathrm{AH}$, juntamente com degradação de compostos mais condensados.

\section{Conclusões}

1. A adição de resíduos orgânicos de origem urbana nas amostras dos solos promove alterações na distribuição das frações humificadas da matéria orgânica; é observada a diminuição da relação $\mathrm{AH} / \mathrm{AF}$, e, como conseqüência, da qualidade do húmus.

2. Ocorrem transformações na distribuição de $\mathrm{C}$ na estrutura dos ácidos húmicos extraídos dos solos com a adição de resíduos decorrentes da alteração do processo de humificação com diminuição no conteúdo de $\mathrm{C}$ alquílico em grupos metilas e metilenos, e aumento de $\mathrm{C}$, atribuído a polissacarídeos. 


\section{Agradecimentos}

A Marcelo Canellas e Márcia Zahur, pela colaboração na realização do trabalho; aos professores Nelson Mazur e Clarice Oliveira, do Departamento de Solos da Universidade Federal Rural do Rio de Janeiro, pela ajuda na obtenção das amostras de solo.

\section{Referências}

BLOOM, P. R.; LEENHEER, J. A. Vibrational, electronic, and high-energy spectroscopic methods for characterizing humic substances. In: HAYES, M. H. B.; MacCARTHY, P.; MALCOLM, R. L.; SWIFT, R. S. (Ed.). Humic substances: II. In search of structure. New York: J. Wiley, 1989. p. $410-446$

CHEN, Y.; SENESI, N.; SCHNITZER, M. Information provided on humic substances by E4/E6 rations. Soil Science Society of America Journal, Madison, v. 41, p. $352-358,1977$

COLTHUP, N. B.; DALY, L. H.; WIBERLEY, S. E. Introduction to infrared and raman spectroscopy. New York: Academic, 1964. 511 p

DABIN, B. Les matières organiques dans les sols tropicaux normalement drainés. Cahiers de l'Office de la Recherche Scientifique et Technique Outre-Mer, Série Pédologie, Paris, v. 16, n. 3/4, p. 197-215, 1981.

EMBRAPA. Centro Nacional de Pesquisa de Solos (Rio de Janeiro, RJ). Manual de métodos de análise de solo 2. ed. Rio de Janeiro, 1997. 212 p.

GARCÉS, N. P. Características actuales del humus en los principales tipos de suelos de Cuba. Nitra Universidad de Agricultura de Nitra, 1987. 200 p. Tesis Doctoral.

GHOSH, K.; SCHNITZER, M. UV and visible absorption spectroscopic investigations in relation to macromolecular characteristics of humic substances. Journal of Soil Science, Oxford, v. 30, p. 735-745, 1979.

KÖBEL-KNABER, I. ${ }^{13} \mathrm{C}$ and ${ }^{15} \mathrm{~N}$ NMR spectroscopy as a tool in soil organic mater studies. Geoderma,Amsterdam, v. 80, p. $243-270,1997$
KONONOVA, M. M. Materia orgánica del suelo: su naturaleza, propiedades y métodos de investigación. Barcelona: Oikos-tau, 1982. $364 \mathrm{p}$

KUMADA, K. Chemistry of soil organic matter. Tokyo: Japan Scientific Societies, 1987. 241 p.

MacCALLISTER, D. L.; CHUIEN, W. L. Organic carbon quantity and forms as influenced by tillage and cropping sequence. Communications in Soil Science and Plant Analysis, New York, v. 31, p. 465-479, 2000.

MAZUR, N. Níquel, chumbo, zinco e cobre em solos que receberam composto de resíduos sólidos urbanos. Viçosa, MG: UFV, 1997. 128 p. Tese de Doutorado.

MORENO, J. L. La materia orgánica en los agrosistemas. Madrid: Ministerio de Agricultura Pesca y Alimentación, 1996. 174 p.

NAKANISHI, K. Infrared absorption spectroscopy Tokyo: Nankondo, 1962. 233 p.

OLIVEIRA, C Avaliação do potencial de contaminação de dois solos agrícolas com lodo de esgoto enriquecido com cádmio, chumbo e zinco. Seropédica: UFRRJ, 1998 186 p. Tese de Doutorado.

ORLOV, D. S. Organic substances of Russian soils Eurasian Soil Science, Moscow, v. 31, n. 9, p. 946-953, 1998.

ORTEGA, F. S. El humus de los suelos de Cuba: I. Suelos derivados de esquistos ácidos. Ciencias de la Agricultura, Havana, v. 17, p. 63-89, 1983.

PRESTON, C. M. Applications of NMR to soil organic matter analysis: history and prospects. Soil Science, Baltimore, v. 161, p. 144-166, 1996.

PRESTON, C. M.; HEMPLING, R.; SCHULTEN, H. R.; SCHNITZER, M.; TROFYMOV, J. A.;AXELSON, D. E. Characterization of organic matter in a forest soil of coastal British Columbia by NMR and pyrolysis-field ionization mass spectrometry. Plant and Soil, Dordrecht, v. 158, p. 69-82, 1994a.

PRESTON, C.; NEWMAN, R. H.; ROTHER, P. Using ${ }^{13} \mathrm{C}$ CPMAS NMR to assess effects of cultivation on the organic matter of particle size fractions in a grassland soil. Soil Science, Baltimore, v. 157, n. 1, p. 26-35, 1994 b.

Pesq. agropec. bras., Brasília, v. 36, n. 12, p. 1529-1538, dez. 2001 
SANTOS, G. de A.; CAMARGO, F. A. O. Fundamentos da matéria orgânica do solo: ecossistemas tropicais e subtropicais. Porto Alegre: Gênesis, 1999. 544 p.

SCHNITZER, M.; PRESTON, C. M. Analysis of humic acids by solution and solid-state carbon-13 nuclear magnetic resonance. Soil Science Society of America Journal, Madison, v. 50, p. 326-331, 1986

SCHULTEN, H. R.; SCHNITZER, M. Chemical model structures for soil organic matter and soils. Soil Science, Baltimore, v. 162, n. 2, p. 115-130, 1997.

SILVERSTEIN, R. M.; BASSLER, G.; MORRIL, T. C. Identificação espectrométrica de compostos orgânicos. Rio de Janeiro: Guanabara-Koogan, 1994. 387 p.

STEVENSON, F. J. Humus chemistry: genesis, composition, reaction. New York: Wiley Interscience, 1994. $443 \mathrm{p}$
VEEMAN, W. S. Nuclear magnetic resonance: a simple introduction to the principles and applications. Geoderma, Amsterdam, v. 80, p. 225-242, 1997.

WILSON, M. A. NMR techniques and applications in geochemistry and soil chemistry. Oxford: Pergamon, 1987. $353 \mathrm{p}$

WILSON, M. A. Soil organic matter maps by nuclear magnetic resonance. Journal of Soil Science, Oxford, v. 35 , p. $209-215,1984$

ZECH, W.; SENESI, N.; GUGGENBERGER, G.; KAISER, K.; LEHMANN, J.; MIANO, T. M.; MILTNER, A.; SCHROTH, G. Factors controlling humification and mineralization of soil organic matter in the tropics. Geoderma, Amsterdam, v. 79, p. 117-161, 1997. 